\title{
PHOSPHATE COMPOUNDS OF THE RABBIT RED BLOOD CELL DURING STORAGE IN ACID CITRATE DEXTROSE (ACD) AND ACD-INOSINE *
}

\author{
By GRANT R. BARTLETT AND A. WILLIAM SHAFER
}

(From The Scripps Clinic and Research Foundation, La Jolla, Calif.)

(Submitted for publication June 8, 1959; accepted September 10, 1959)

Red blood cells of the rabbit appear to react about the same as those of the human to storage in acid citrate dextrose (ACD) preservative in the cold; the cells of both species show a posttransfusion survival of approximately 70 per cent at three weeks and less than 50 per cent at six weeks. The fall in survival is accompanied by a reduction in the glycolytic rate and in the organic phosphate content and these changes are retarded in the presence of certain nucleosides (1-3). These facts suggest that rabbit blood may be substituted for human blood in the study of certain problems related to the maintenance of red cell viability during storage.

The mechanism responsible for the loss of red cell viability during storage is not known, but one reasonable hypothesis would implicate a disturbance in the cell's carbohydrate metabolism. To learn more about the state of the metabolic machinery, which is predominantly glycolytic, rabbit blood was kept in ACD at $4^{\circ} \mathrm{C}$. with and without inosine and the phosphorlylated metabolic intermediates of the red cells were analyzed by ionexchange chromatography at the beginning and after three and six weeks of storage.

\section{METHODS}

Blood was collected by heart puncture with heparinized syringes from female New Zealand strain rabbits, 2 to 3 $\mathrm{Kg}$. in weight, and was mixed with Formula B (National Institutes of Health) ACD solution. Four mg. of inosine-which had been autoclaved in normal salineper ml. of blood, was added to half of the pooled blood mixture. One hundred $\mathrm{ml}$. aliquots of the blood mixture were removed for analysis initially and after three and six weeks of storage at $4^{\circ} \mathrm{C}$. Aseptic precautions were followed throughout the experimental manipulations and there was no evidence of bacterial contamination at the end of the storage period.

* Supported in part by the U. S. Army Medical Research and Development Command, Office of the Surgeon General, and the San Diego Heart Association.
The aliquot of blood was centrifuged, the plasma and buffy coat withdrawn, and the packed red cells were suspended in two volumes of normal saline and recentrifuged. The washed red cells were weighed, the small amount of saline present being measured by a hematocrit determination, and then were mixed thoroughly with two volumes of 10 per cent trichloroacetic acid and the suspension centrifuged. The residue was extracted with the same volume of 5 per cent trichloroacetic acid. All operations to this point were performed at $0^{\circ}$ to $5^{\circ} \mathrm{C}$. The acid was removed by extracting with two volumes of ether four times.

The red cell extract was neutralized with $1 \mathrm{~N}$ ammonium hydroxide, diluted fourfold and passed through a $1 \times 15 \mathrm{~cm}$. column of Dowex $1-\times 8$ chloride (60 to 80 wet mesh) which was eluted at $2.5 \mathrm{ml}$. per minute with solutions of hydrochloric acid and ammonium chloride, as shown in Figures 1 and 2. Fractions of $20 \mathrm{ml}$. volume were collected and each was assayed for optical density at $260 \mathrm{~m} \mu$ and for total phosphorus. The $\mathrm{HCl}$ elution fractions were neutralized on the day of collection. The compounds were identified from their elution position in the chromatograph as determined with standards and with the more completely characterized components of chromatographed extracts of fresh rabbit blood drawn into heparin. The identity of the nucleotide peaks was verified by their ultraviolet spectra and by the phosphorus and ribose [orcinol reaction (4)] content. The $0.01 \mathrm{~N} \mathrm{HCl}$ and $0.1 \mathrm{~N} \mathrm{NH}_{4} \mathrm{Cl}$ sections of the chromatograph were tested for glucose and fructose mono- and diphosphates by the anthrone (5) and carbazole (6) methods. Sedoheptulose-7-phosphate was identified and quantified by its characteristic reaction in the cysteinesulfuric acid method (7) as well as by the distinctive colors which it produced in the carbazole, anthrone and orcinol tests. In addition, its elution position was compared with an authentic sample of sedoheptulose-7-phosphate which had been prepared from ribose-5-phosphate with spinach transketolase (8).

For rechromatography, the chloride eluates were diluted fourfold and passed through $1 \times 15 \mathrm{~cm}$. columns of Dowex $1-\times 8$ formate (100 to 325 wet mesh) which were eluted at 0.5 to $1.0 \mathrm{ml}$. per minute with linearly increasing concentrations of formic acid or ammonium formate buffers as indicated in the figures. Further details concerning the procedures which were used for the extraction of the red cells, for ion-exchange chromatography and for the identification of the individual compounds, are given elsewhere (9-12). 


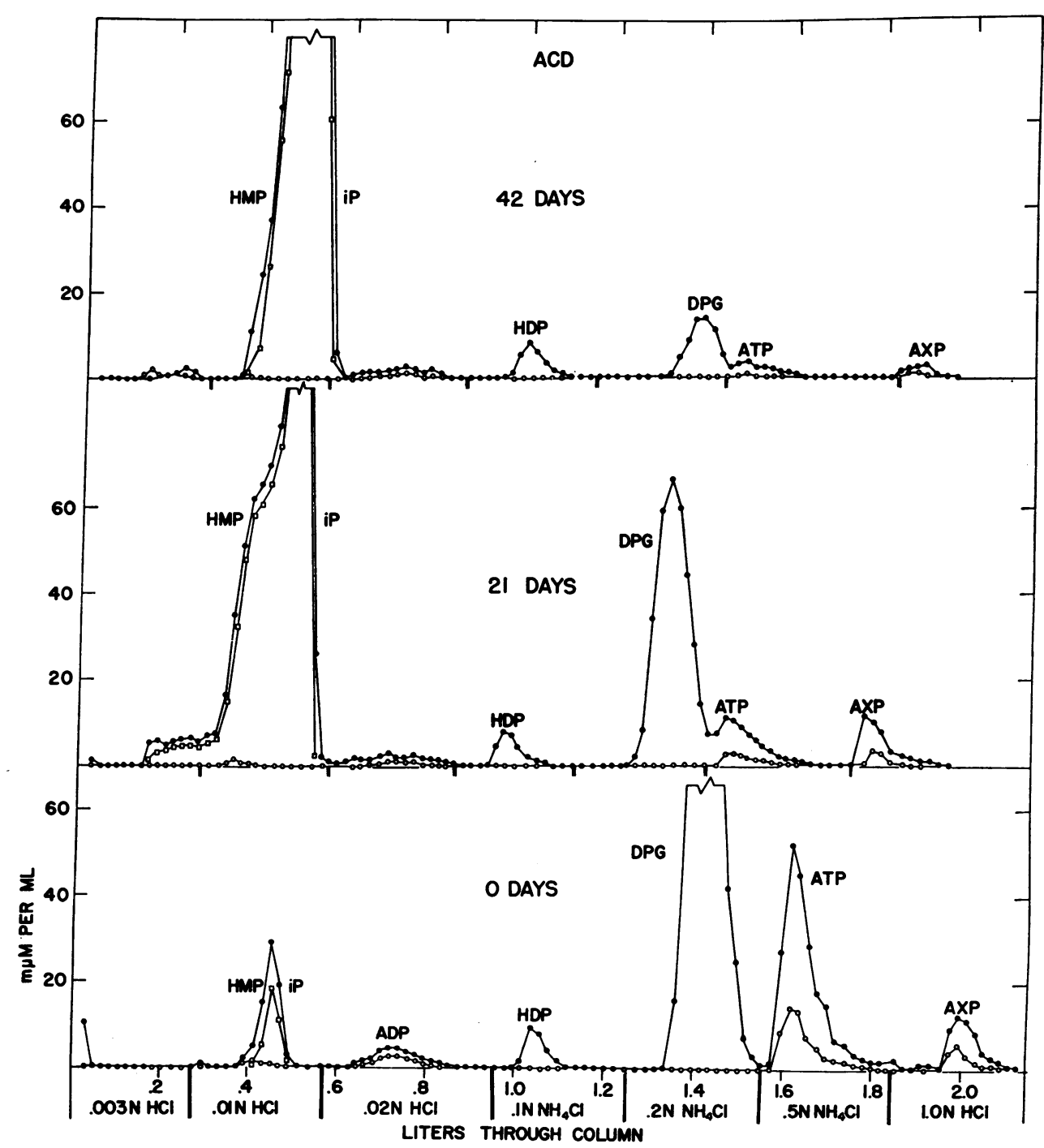

Fig. 1. Ion-Exchange Chromatographs of Trichloroacetic Acid Extracts of Rabbit Red Cells Stored in Acid Citrate Dextrose (ACD) for 0, 21 and 42 Days

The data are plotted in millimicromoles per milliliter of eluate (calculated for $1 \mathrm{ml}$. of red cells) and refer to: total phosphorus, $\bullet$; adenine (from the optical density at $260 \mathrm{~m} \mu$ ), $\bigcirc$; and inorganic phosphate, $\square$. The paired vertical lines on the three abscissae indicate where the eluting agent was changed. Abbreviations: HMP, hexose and heptose monophosphate; IP, inorganic phosphate; ADP, adenosine diphosphate; HDP, hexose diphosphate; DPG, 2,3diphosphoglycerate; ATP, adenosine triphosphate; AXP, unknown nucleotide.

\section{RESULTS AND DISCUSSION}

Figures 1 and 2 summarize the results of the ion-exchange analyses of the rabbit red cells which had been stored for zero, three and six weeks in $A C D$ and in ACD plus inosine. The overall picture is one of a marked shift of organic to inorganic phosphate with some significant differences in the pattern in the presence of inosine.
The fall in the level of adenosine triphosphate (ATP) was not associated with any increase in adenosine monophosphate (AMP) or adenosine diphosphate (ADP), probable intermediates.

Since a shift in the ultraviolet absorption maximum was found in the ADP area of some of the chloride chromatographs, these fractions were rerun on Dowex-formate. Figure 3 shows that 


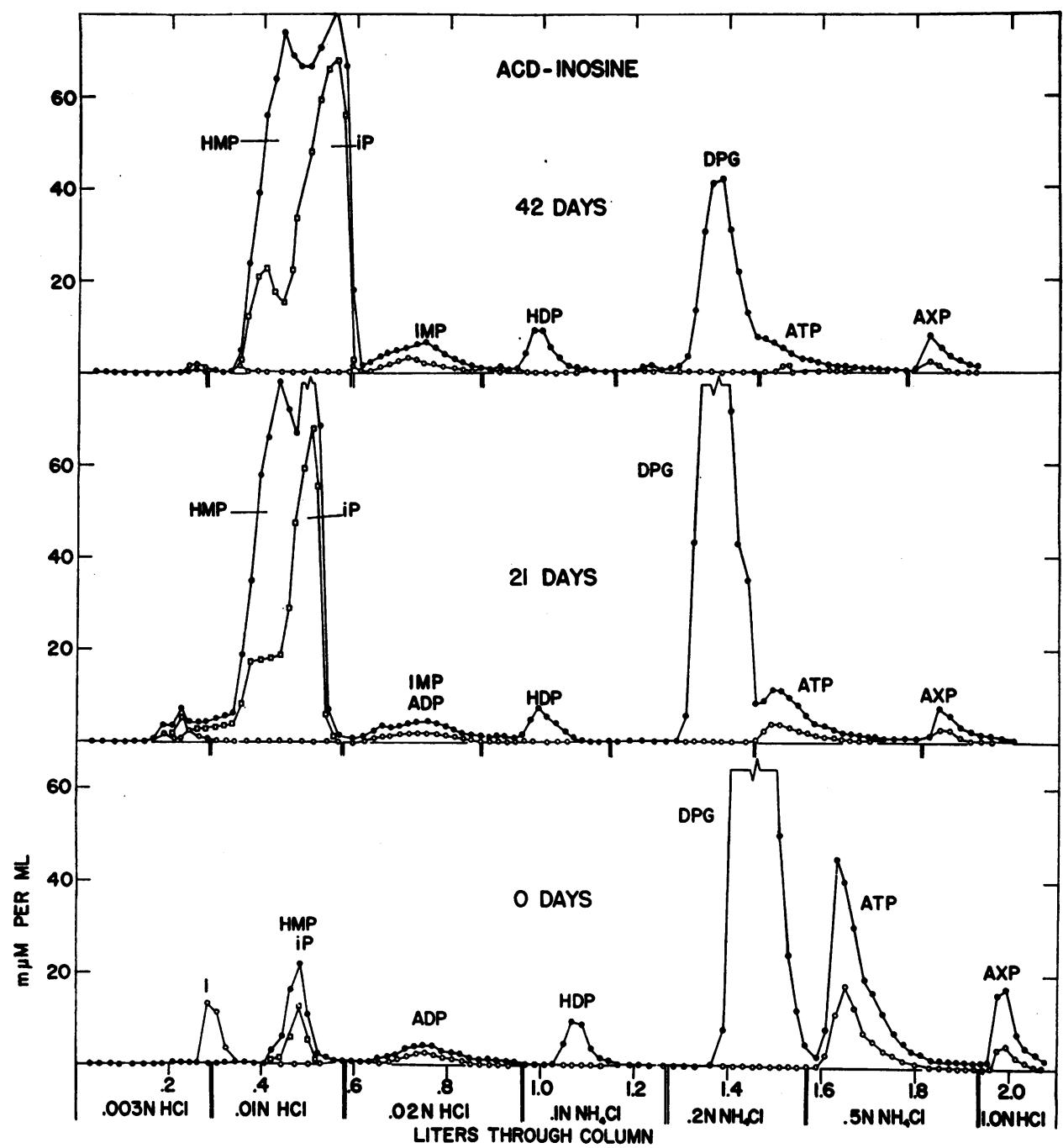

Fig. 2. Ion-Exchange Chromatographs of Trichloroacetic Acid Extracts of Rabbit Red Cells Stored in ACD Plus Inosine for 0, 21 and 42 Days

See Figure 1. IMP, inosinic acid; I, inosine.

the material consisted predominantly of a mixture of inosinic acid and ADP. It is seen that the loss of ADP was slow in the first three weeks but was accelerated in the second with some sparing effect of inosine. Of interest was the increasing concentration of inosinic acid in the cells stored with inosine. Figure 3 includes a standard chromatograph which shows the elution characteristics of known inosinic mono- and diphosphates and of ADP.

Despite the large quantity of 2,3-diphosphoglycerate (DPG) being decomposed in the blood during storage, there was no evidence for the accumulation of any monophosphoglycerate, phos- phopyruvate, pyruvate or glyceric acid, either in the red cells or in the plasma. Presumably the DPG was split to 2-monophosphoglycerate by a phosphatase and then further degraded through phosphopyruvate and pyruvate to lactic acid, the intermediates being metabolized as rapidly as formed.

The hexosediphosphate peak in a chromatograph of normal red cells from blood drawn into heparin includes a mixture of fructose 1,6-diphosphate and glucose 1,6-diphosphate in a ratio of about four to one (13). For some unexplained reason, the fructose diphosphate rapidly disap- 
peared from the blood which was collected in ACD or in ACD-inosine. In contrast to the lability of the fructose diphosphate, glucose diphosphate remained stable throughout the storage period in both incubation media. In view of these unusual reactions, it was considered worthwhile to perform additional tests to verify the identity of the glucose diphosphate. An extract of $75 \mathrm{ml}$. of rabbit red cells, collected in $\mathrm{ACD}$, was chromatographed on a column of Dowex-1 with chloride eluants as described above. The peak in the 0.1 $\mathrm{NH}_{4} \mathrm{Cl}$ elution section gave the anthrone and phosphorus results required for glucose diphosphate and was negative for fructose diphosphate (carbazole test). The combined fractions were treated with Dowex-50 to remove ammonium ions and the resulting $0.1 \mathrm{~N} \mathrm{HCl}$ solution was heated for 10 minutes at $100^{\circ} \mathrm{C}$, a procedure which would be expected to hydrolyze the glucose diphosphate at the 1 position without affecting the 6. Figure 4 shows that the acid hydrolysis produced a mixture of equal parts of glucose-6-phosphate and inorganic phosphate. The glucose-6phosphate was in the correct elution position, had equivalent anthrone (as hexose) and total phosphorus values and gave approximately an equivalent reduction of triphosphopyridine nucleotide (TPN) in the glucose-6-phosphate dehydrogenase assay system. This provides additional support for the conclusion that the substance was in fact glucose 1,6-diphosphate.

The normal red blood cell of the rabbit had been found to contain only a very small concentration of the hexose monophosphates, glucose-6phosphate and fructose-6- phosphate (13). The hexosemonophosphate elution section of the chro-
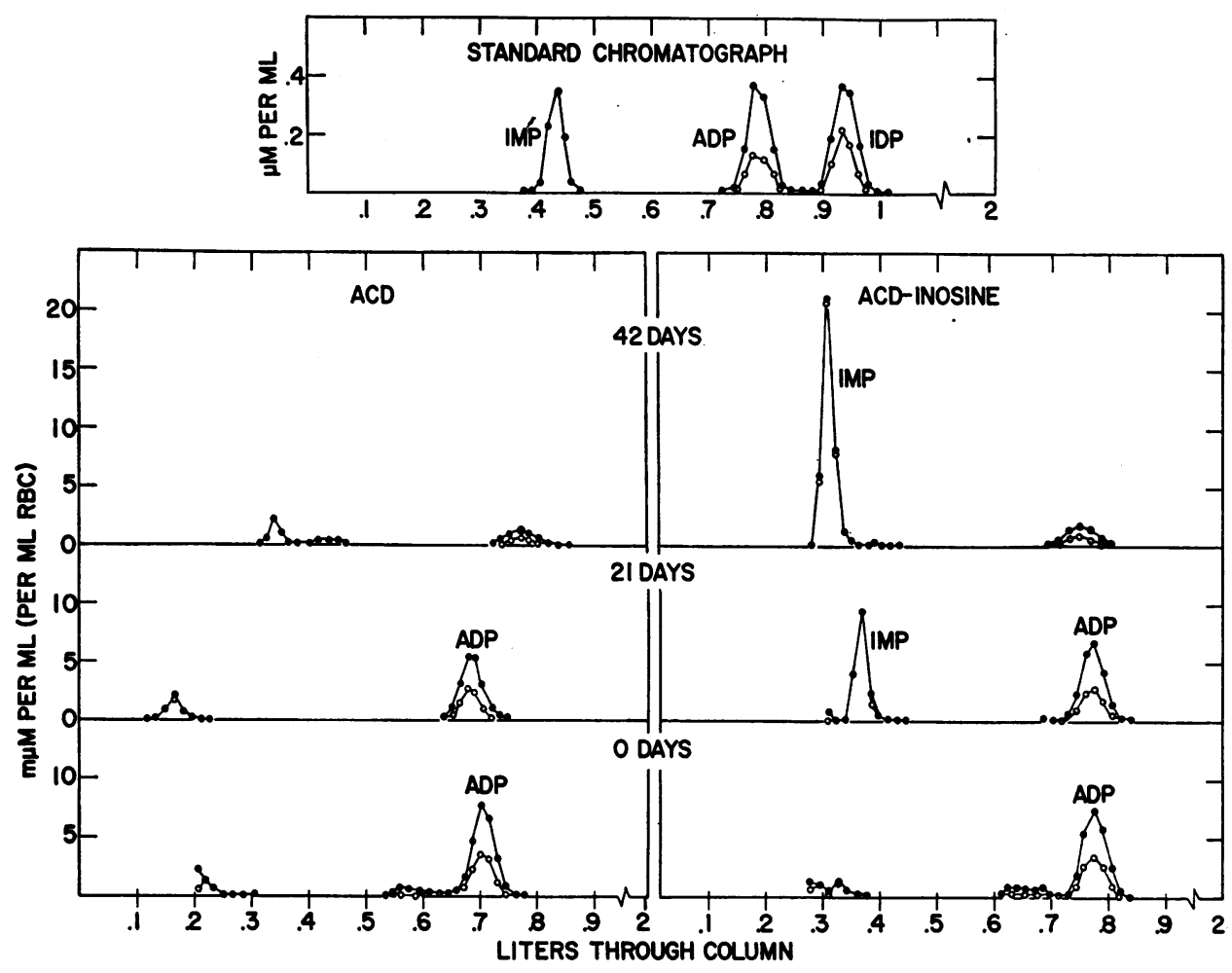

Fig. 3. Rechromatographs of the $0.02 \mathrm{~N}$ HCl Sections of the Dowex Chloride ChroMatographs Shown in Figures 1 and 2

The pooled chloride eluates were passed through $1 \times 15 \mathrm{~cm}$. columns of Dowex 1- $\times 8$ formate (100 to 325 wet mesh) which were eluted at $1 \mathrm{ml}$. per minute with $2 \mathrm{~L}$. of linear gradient zero to $2 \mathrm{~N}, \mathrm{pH} 3.6$, ammonium formate. Five $\mu$ Moles of each compound was used in the standard chromatograph. The data are plotted in millimicromoles per milliliter of eluate (calculated for $1 \mathrm{ml}$. of red cells) and refer to: total phosphorus, ; and adenine (from the optical density at $260 \mathrm{~m} \mu$ ), O. For abbreviations, see Figures 1 and 2; IDP, inosine diphosphate. 


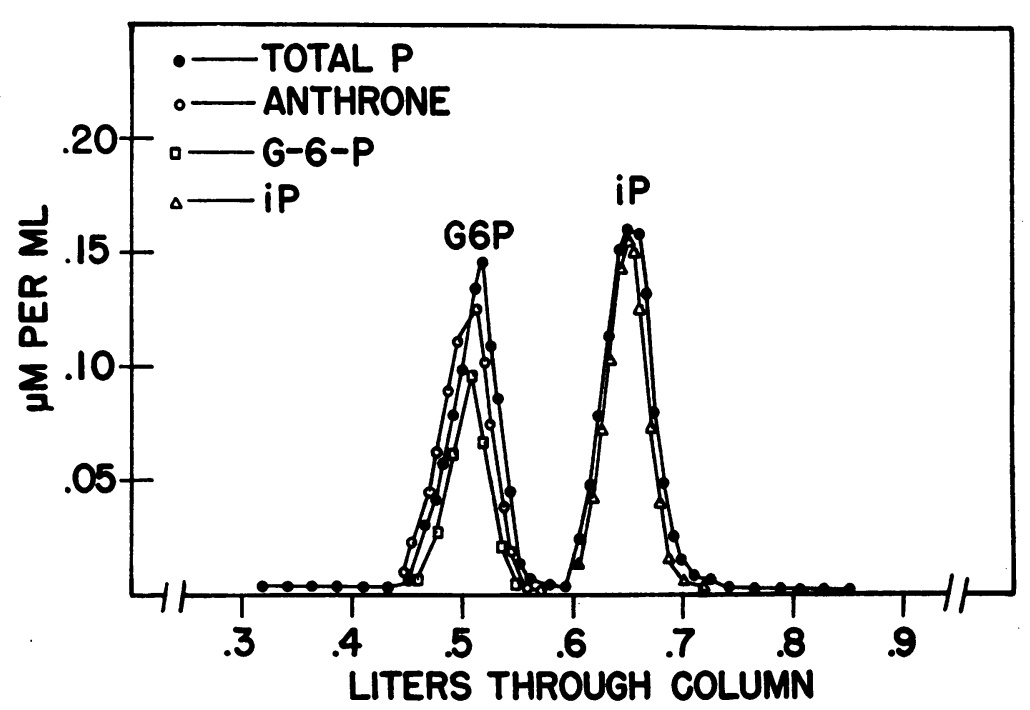

Fig. 4. Chromatograph of Acid Hydrolysis Products of Glucose Diphosphate from Rabit Red Cells Collected in ACD

The acid hydrolysis products of the HDP peak of a chloride chromatograph were passed through a $1 \times 15 \mathrm{~cm}$. column of Dowex $1-\times 8$ formate (100 to 325 wet mesh) which was eluted at $1 \mathrm{ml}$. per minute with $2 \mathrm{~L}$. of linear gradient zero to $1 \mathrm{~N}$ formic acid. $\square$, G6P (glucose-6-phosphate) dehydrogenase assay.

matograph of the rabbit red cells which were stored in ACD alone gave a weak reaction in the anthrone and carbazole tests. With ACD-inosine, however, an intense color was obtained in the two tests at three and six weeks of storage, with an anthrone reaction atypical of hexoses. Further study disclosed that these fractions also gave a strong reaction typical of ketoheptose with the crysteine-sulfuric acid reagent. To obtain more information about the contents of the 0.003 and $0.01 \mathrm{~N} \mathrm{HCl}$ elution sections, all fractions of the three week control and of the three and six week inosine experiments were pooled and rechromatographed on columns of Dowex-1 formate using gradient elution with zero to $1 \mathrm{~N}$ formic acid. The results from the three week control confirmed the tests which had been carried out in the chloride chromatography: the organic phosphate consisted predominantly of small amounts of glucose-6phosphate and sedoheptulose-7-phosphate. The three and six week inosine chromatographs were qualitatively the same and the six week data are presented in Figure 5. Almost all of the organic phosphate was eluted as a single peak well in advance of the inorganic phosphate, had the same chromatographic location, and gave the same colors in the cysteine-sulfuric, carbazole, orcinol and anthrone reactions as did a sample of purified sedoheptulose-7-phosphate prepared from ribose-5phosphate with spinach extract. There was a slight excess of anthrone and phosphorus reacting substance as compared to cysteine-sulfuric and carbazole in the later eluting end of the curve, suggesting the presence of a small amount of glucose6-phosphate. The section of the chromatograph between the sedoheptulose-7-phosphate and the inorganic phosphate would be occupied by ribose-5-phosphate and the ketopentose-5-phosphates if they had been present. This area was negative for phosphorus and orcinol demonstrating that these expected pentose shunt intermediates of inosine metabolism must have been present in exceedingly small concentrations.

The amounts of some of the phosphate esters which were found at the three storage periods are summarized in Figure 6 . The effect of inosine on the maintenance of the organic phosphates of the red cell was not, in some respects, as great as or of a kind that had been anticipated from previous studies (1-3), although most of these had been 
performed by incubating the blood at $38^{\circ} \mathrm{C}$. with adenosine or inosine at some stage of the storage, and so are not directly comparable. In the present experiments inosine had little influence on the breakdown of ATP. Although there was two or three times as much DPG in the inosine-treated blood at three and six weeks, nevertheless, a major fraction of this compound had been decomposed in both systems. The most striking effect of the inosine was in bringing about a large accumulation of sedoheptulose-7-phosphate at three and six weeks in the apparent absence of ribose, ribulose and xylulose phosphates.

An earlier investigation of the changes of the phosphate compounds of the human red cell during storage in ACD showed a somewhat different pattern of breakdown (14). The DPG had dropped to very low levels at two weeks, at which time there was only a relatively small loss of ATP. The rate of disappearance of the ATP was then accelerated with more prolonged storage. As with

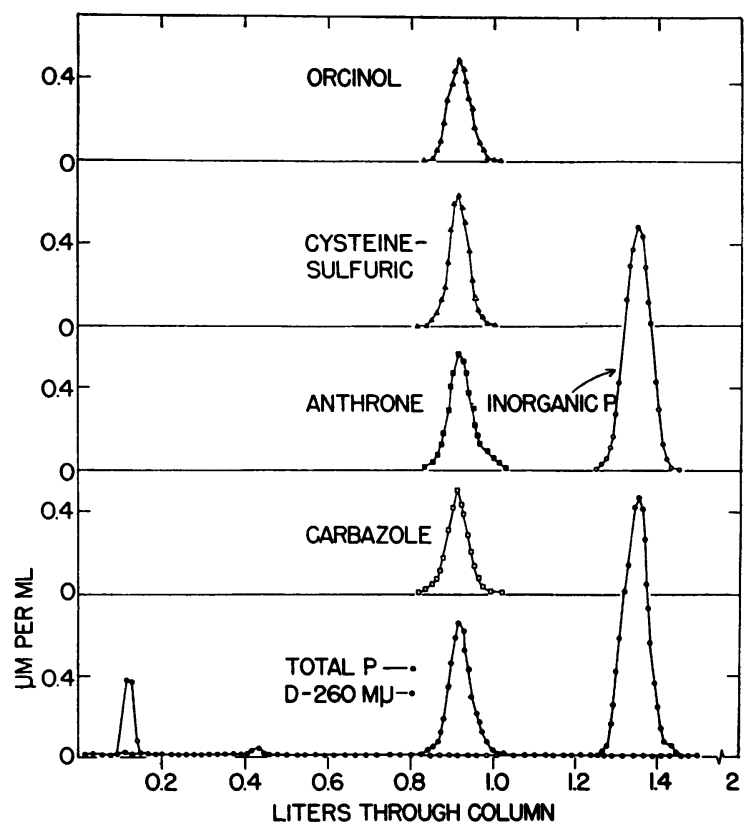

Fig. 5. Rechromatograph of the 0.003 and $0.01 \mathrm{~N}$ HCl Sections of the Chloride Chromatograph (Figure 2) of Rabbit Red Cells Stored Six Weeks in ACD Plus Inosine

A $1 \times 15 \mathrm{~cm}$. column of Dowex $1-\times 8$ formate (100 to 325 wet mesh) was eluted at $1 \mathrm{ml}$. per minute with $2 \mathrm{~L}$. of linear gradient zero to $1 \mathrm{~N}$ formic acid. First small ultraviolet absorbing peak, inosine; second peak, sedoheptulose-7-phosphate; third peak, inorganic phosphate.

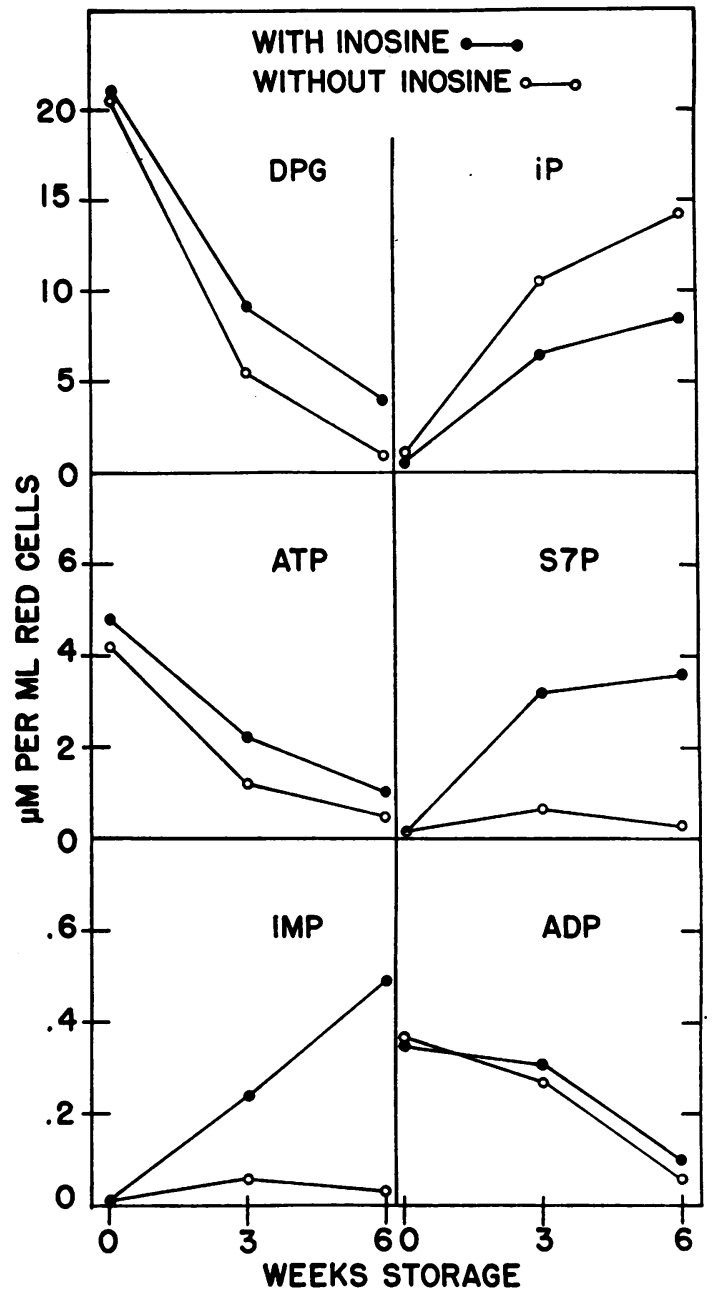

Fig. 6. Changes of Phosphorus Compounds of the Rabbit Red Cell during Cold Storage in ACD and in ACD Plus Inosine

The values are given in micromoles of phosphorus per milliliter of red cells. For abbreviations, see Figures 1 and 2; S7P, sedoheptulose-7-phosphate.

the rabbit, the fructose diphosphate of the human red cell disappeared on mixing the blood with ACD while the glucose diphosphate remained elevated throughout most of the storage.

The profound chemical changes taking place in the rabbit red cell during storage in ACD which are recorded here do not yet provide specific answers as to the nature of the metabolic defect, its relationship to red cell viability and the influence of inosine. This new information obtained, however, should prove significant to an eventual solution of these problems. 


\section{SUM MARY}

The water-soluble phosphate compounds of the rabbit red blood cell have been assayed by ion-exchange chromatography at zero, three and six weeks of storage at $4^{\circ} \mathrm{C}$. in acid citrate dextrose (ACD) and ACD-inosine preservative solutions. There was a marked shift of organic to inorganic phosphate during the storage brought about chiefly by the breakdown of 2,3-diphosphoglycerate and adenosine triphosphate, a process which was retarded slightly in the presence of inosine. Adenosine diphosphate showed only a slight drop at three weeks but had almost disappeared at six, again with some slowing of the breakdown given by inosine. Fructose diphosphate disappeared from the red cells immediately upon mixing with ACD, but glucose diphosphate did not change throughout the storage period in either media. There was a relatively large increase in inosinic acid with inosine at three and six weeks. The most striking effect of the inosine was that it produced a large amount of sedoheptulose-7-phosphate at three and six weeks in the absence of a significant accumulation of other pentose shunt intermediates.

\section{REFERENCES}

1. Gabrio, B. W., Finch, C. A., and Huennekens, F. M. Erythrocyte preservation: A topic in molecular biochemistry. Blood 1956, 11, 103.

2. Gabrio, B. W., Donohue, D. M., and Finch, C. A. Erythrocyte preservation. V. Relationship between chemical changes and viability of stored blood treated with adenosine. J. clin. Invest. 1955, 34, 1509.

3. Donohue, D. M., Finch, C. A., and Gabrio, B. W. Erythrocyte preservation. VI. The storage of blood with purine nucleosides. J. clin. Invest. 1956, 35, 562.

4. Mejbaum, W. Über die Bestimmung kleiner Pentosemengen, insbesondere in Derivaten der Adenylsäure. Z. physiol. Chem. 1939, 258, 117.

5. Dreywood, R. Qualitative test for carbohydrate material. Industr. engin. Chem., Analyt. ed., 1946 18, 499.

6. Dische, Z., and Borenfreund, E. A new spectrophotometric method for the detection and determination of keto sugars and trioses. J. biol. Chem. 1951, 192, 583 .

7. Dische, Z. Qualitative and quantitative colorimetric determination of heptoses. J. biol. Chem. 1953, 204, 983.

8. Horecker, B. L. Preparation and analysis of heptulose phosphates in Methods in Enzymology, S. P. Colowick and N. O. Kaplan, Eds. New York, Academic Press Inc. 1957, vol. III, 195.

9. Bartlett, G. R. Human red cell glycolytic intermediates. J. biol. Chem. 1959, 234, 449.

10. Bartlett, G. R. Methods for the isolation of glycolytic intermediates by column chromatography with ion exchange resins. J. biol. Chem. 1959, 234, 459.

11. Bartlett, G. R. Phosphorus assay in column chromatography. J. biol. Chem. 1959, 234, 466.

12. Bartlett, G. R. Colorimetric assay methods for free and phosphorylated glyceric acids. J. biol. Chem. 1959, 234, 469.

13. Bartlett, G. R. Unpublished data.

14. Bartlett, G. R., and Barnet, H. N. Changes in the phosphate compounds of the human red blood cell during blood bank storage. J. clin. Invest. 1960, 39, 56. 